\title{
La Convención Internacional sobre los derechos de las personas con discapacidad y las teorías de la justicia: La participación política de las personas con discapacidad
}

\section{The International Convention on the Rights of Persons with disabilities and theories of justice political participation of people with disabilities}

\section{Elkin Andrés Heredia Ríos}

\author{
Magíster en Estudios Políticos
}

Fundación Universitaria Autónoma de las Américas, Pereira - Colombia

Correo electrónico: elkin.heredia@uam.edu.co

\author{
Alexandra Hurtado Maya \\ Magíster en Educación desde la Diversidad", Universidad de Manizales \\ Fundación Universitaria Autónoma de las Américas, Pereira - Colombia \\ Correo electrónico: alexandra.hurtado@uam.edu.co \\ Jenny Alexandra Echeverri Herrera (D) \\ Magíster en Filosofía \\ Universidad Libre Seccional Pereira. Pereira -Colombia \\ Correo electrónico: jennya.echeverrih@unilibre.edu.co
}




\section{Resumen}

Partiendo de los principios sociales de la Convención Internacional de Naciones Unidas sobre los derechos de las personas con discapacidad (2006), se estudia el modelo social de la discapacidad que la inspiró, con el propósito de evaluar los modelos de justicia que pueden llegar a ajustarse a esta transformación en la comprensión de la discapacidad. Paso seguido se analizan las teorías de la justicia más relevantes que se han diseñado en las últimas décadas a partir del criterio de la manera en que abordan el problema de la justicia para las personas con discapacidad. Finalmente, se propone un balance de las teorías expuestas y se toma una posición relacionada con la demanda de participación política que se expresa en la Convención y en el informe sobre su implementación en Colombia en el año 2016.

\section{Palabras clave}

Discapacidad; Justicia; Política; Derechos; Reconocimiento; Derecho Internacional.

\section{Abstract}

Based on the social principles of the United Nations International Convention on the Rights of Persons with Disabilities (2006), we study the social model of disability that inspired it to evaluate the models of justice that can be adjusted to this transformation in the understanding of disability. Next, we analyze the most relevant theories of justice designed in recent decades based on the criteria of how they approach the problem of justice for people with disabilities. Finally, we expose a balance of the theories. We proposed a position related to the demand for political participation expressed in the Convention and the report on its implementation in Colombia in 2016.

\section{Keywords}

Disability; Justice; Politics; Rights; Recognition; International right

\section{Cómo citar este artículo:}

Heredia Ríos, E., Hurtado Maya, A., \& Echeverri Herrera, J. (2021). La Convención Internacional sobre los derechos de las personas con discapacidad y las teorías de la justicia: La participación política de las personas con discapacidad. Revista de la Facultad de Derecho y Ciencias Políticas, 51 (134), pp. $1-24$.

doi: https://doi.org/10.18566/rfdcp.v51n134.a01

Recibido: 25 de noviembre de 2019

Aprobado: 29 de septiembre de 2020 


\section{Introducción \\ El modelo social de la discapacidad y las teorías de la justicia}

De acuerdo con datos de la Organización Mundial de la Salud (OMS) (2017) alrededor del $15 \%$ de la población mundial (más de 1000 millones) presenta algún tipo de discapacidad. El 80 \% de estas personas viven por debajo de la línea de la pobreza. En razón de causas medioambientales, precariedad en los sistemas de salud, crecimiento descontrolado de las grandes capitales, esta cifra probablemente aumentará (OMS, 2017) En Colombia, según datos proporcionados por el Ministerio de Salud y Protección Social en el año 2017, existen un total de 1.342.222 personas en situación de discapacidad, el $13 \%$ son víctimas del conflicto armado (172 503), el 30 \% no tienen ningún tipo de escolaridad, y el $80 \%$ pertenecen a los estratos socioeconómicos 1 y 2 . De la misma manera, el $80 \%$ de las personas que han logrado tener un trabajo lo hacen sin ningún tipo de contrato formal, y solo una mínima parte (1\%) accede a la universidad (Ministerio de Salud y Protección Social de Colombia, 2017, pp.8-13). Estos datos hablan de algo que una simple constatación, en la realidad, permite comprobar: las personas con discapacidad $(\mathrm{PcD})$ viven en un mundo en contravía con sus particularidades y necesidades específicas.

Solo hasta después de la Segunda Guerra Mundial se ha creado consciencia en torno a la necesidad de un trato justo, igualitario, y que promueva el ejercicio de la autonomía para este grupo importante de personas (Palacios y Bariffi, 2007). Tras algunos acercamientos que partían del respeto a la dignidad de las personas y a la igualdad de todos los seres humanos después de los años cincuenta, se llega finalmente en el siglo XXI a consolidar una legislación integral y verdaderamente incluyente para las PcD (MartínezRozo, Uribe-Rodríguez, Velásquez-González, 2014). La Asamblea General de la $\mathrm{ONU}$, el 13 de diciembre de 2006, promulga la Convención Internacional sobre los Derechos de las Personas con Discapacidad, una conquista realmente importante para ese $15 \%$ de la población.

Los principios sobre los que sustentó la Convención -expuestos de una manera sintética - son los siguientes: 1. Dignidad de la persona y, por ende, autonomía e independencia; 2. Igualdad: a). como no discriminación, b). como igualdad de oportunidades, c). como igualdad de género y d). como participación en la toma de decisiones colectivas; 3. Respeto e inclusión de 
la diversidad. El propósito de la Convención fue “(...) proteger y asegurar el goce pleno y en condiciones de igualdad de todos los derechos humanos y libertades fundamentales por todas las personas con discapacidad, y promover el respeto de su dignidad inherente” (ONU, 2006, Artículo 1) . Estos principios y propósitos sustentaron una definición de la discapacidad fundamentada en el reconocimiento de la multiculturalidad, las contingencias históricas y las relaciones de poder que subyacen en cualquier interacción social (Foucault, 2010a). Es por esto que en la convención se afirma que: "La discapacidad es un concepto que evoluciona y que resulta de la interacción entre las personas con deficiencias y las barreras debidas a la actitud y al entorno que evitan su participación plena y efectiva en la sociedad, en igualdad de condiciones con las demás” (ONU, 2006, Preámbulo). De modo que la Convención entiende la necesidad de tomar la definición de discapacidad a partir del reconocimiento de los factores históricosociales que afectan a cualquier ser humano, pero, muy especialmente, a las PcD.

Como lo ha señalado una amplia literatura reciente (Palacios, 2008; Asís Roig, 2006; Palacios, Bariffi, 2007; Barton, 1998), se ha pasado en materia del reconocimiento de derechos de las $\mathrm{PCD}$ de un enfoque apoyado en la asistencia y el cuidado a un enfoque centrado en los derechos, inspirado por una concepción social de la discapacidad. Se han dejado atrás los dos modelos que diseñaron gran parte de las políticas públicas del pasado, el de la prescindencia y el rehabilitador, que veían en la discapacidad una desviación biológica o un condicionante para poder alcanzar una vida plena. Desde una concepción de la discapacidad que se definía principalmente por las deficiencias, entendidas como las diversas pérdidas en las funciones psicológicas, fisiológicas o anatómicas (Padilla-Muñoz, 2010), se pretendió incluir a las PcD a través del aseguramiento de la movilidad, el acceso a los sistemas de salud, la garantía de un cuidado y asistencia. Pero, a través de estas medidas, se negaba la necesidad de independencia y autonomía que las mismas PcD exigían.

Este hecho produjo en los años setenta una respuesta en el ámbito estadounidense, con repercusiones posteriores en el resto del mundo, como fue la creación del Movimiento de Vida Independiente (Palacios, 2008). Inspirado por los cambios culturales que se suscitaron en la década anterior, buscaba la inclusión radical por medio de demandas en torno a la independencia, autonomía e igualdad. Lo central para este movimiento no era dilucidar la causa de la discapacidad, sino desentrañar las maneras en que la sociedad imperante se había estructurado; es decir, de lo que se trataba era de hacer ver las determinaciones sociohistóricas que convertían a una persona en discapacitada. 
La discapacidad, entonces, se define por los factores que condicionan la forma en que los individuos se perciben a sí mismos y por la manera en que la sociedad los define y aprecia: “(...) la discapacidad estaría compuesta por los factores sociales que restringen, limitan o impiden a las personas con diversidad funcional, vivir una vida en sociedad" (Palacios, 2008, p.123).

Como efecto de este movimiento y de las múltiples luchas emprendidas por organizaciones no gubernamentales y por las propias $\mathrm{PcD}$, se pasó a entender la discapacidad como una forma de opresión social (Young, 2000). De igual manera, se cuestionó el concepto de "normalidad” a través de la discusión de su carácter regulador de la conducta y de las relaciones de poder que suponen su constitución, tal y como lo muestra Foucault en el emblemático Vigilar y castigar (2010b). En conclusión, el modelo social que surge con el Movimiento de Vida Independiente obliga a entender la discapacidad y la justicia en términos de inclusión, autonomía y participación, pero no en términos de asistencia, cuidado, tratamiento, etc., porque resultan contrarios a la autonomía, la dignidad y la igualdad.

La Convención de 2006 adoptó este enfoque de manera explícita, y en consecuencia ha planteado la necesidad de que los países que se han adherido, tal y como lo hizo Colombia en el 2013 (Ley Estatutaria 1618), sustenten su interés por garantizar la realización de una vida buena por parte de las PcD en los siguientes principios: a). dignidad de las personas, b). autonomía y c). interdependencia.

Ahora bien, tras estas transformaciones desde lo jurídico y político resulta importante preguntar por el tipo de modelo de justicia social que puede ser el más indicado para acercarse a la discapacidad como un fenómeno social. La justicia social guarda una estrecha relación con la estructuración del orden sociopolítico, y es por eso que las PcD, al ser un grupo significativo de las sociedades contemporáneas, merecen y exigen una consideración desde el punto de vista de si son o no reconocidas sus demandas como demandas de justicia.

Los modelos disponibles de justicia social se han dividido en dos grandes grupos: redistribución y reconocimiento (Fraser, Honneth, 2006). Aunque se puede añadir un tercer grupo, que ve en la justicia la posibilidad de la transformación social (Young, 2000; Fraser, 2010 Mouffe, 2012).

Entonces, teniendo presente la concepción social de la discapacidad de la Convención: ¿cuál teoría de la justicia resulta ser la más conveniente para 
las PcD? En lo que se sigue se intentará dar respuesta a este interrogante examinando las teorías de justicia más importantes y divergentes entre sí que se encuentran disponibles. El trabajo de revisión consistió en explorar las teorías de la justicia en torno a lo que plantean sobre la discapacidad. Se analizó la categoría de "discapacidad" en cuatro teorías discutidas mayoritariamente en la literatura disponible. Finalmente, se tomará una postura en relación con la conveniencia de alguna concepción de justicia según la necesidad de correspondencia con el modelo social de la discapacidad y el informe sobre los avances en la implementación que hizo las Naciones Unidas en 2016.

\section{Las teorías de la justicia y la discapacidad}

\section{Rawls: la justicia como exclusión de la discapacidad}

Es John Rawls quien, con su monumental trabajo Teoría de la justicia (1971) vuelve a traer al centro del debate el problema de la justicia. Lo hace continuando con la tradición que, desde Aristóteles, pasando por David Hume y el utilitarismo, entiende la justicia en términos redistributivos (Grueso, 2010). La redistribución parte de la realidad de un mundo desigual en el que las cargas y los beneficios no se encuentran repartidos de manera equitativa (Rawls, 2012). Para que pueda darse la unanimidad es necesario que los principios de la justicia no se funden sobre concepciones comprehensivas, sino solo sobre argumentos que cualquier persona, independientemente de su condición moral o religiosa, podría aceptar. Esto último es la idea de un liberalismo político que se mueve como telón de fondo de toda la propuesta (Rawls, 1996).

Amartya Sen (2014) y Martha Nussbaum (2007) han puesto el dedo en la llaga, puesto que, a la hora de considerar el problema de las PcD, Rawls parece no prestarle mucha importancia a este grupo de personas. Es decir, que al aplazar el tema de la discapacidad excluye de la construcción de los principios de justicia a las PcD, y así termina por proponer una teoría que estaría en contravía con los principios que este grupo social ha logrado conquistar a través de las luchas sociales.

De acuerdo con esta concepción de las personas, Rawls omite de la situación de elección política básica las formas más extremas de necesidad y dependencia que pueden experimentar los seres humanos, ya sean físicas o mentales, y ya sean permanentes o temporales. No se trata de ningún descuido, sino de un diseño deliberado. (Nussbaum, 2007, p.121) 
Rawls -y en este punto se sigue la argumentación de Nussbaum (2007)restringe el principio de la dignidad, radicaliza la dependencia y promueve el individualismo por medio de una idea de cooperación como beneficio individual. Las razones de este problema en la teoría de Rawls son dos: a). Su adhesión a la tradición del contrato social sin cuestionar varios de sus principios problemáticos, en especial, el de la naturaleza de los miembros de la situación original, y b). su propuesta de otorgar unos bienes primarios universales (libertades básicas, libertad de movimiento y de elección profesional, poderes y prerrogativas, ingresos y riqueza, bases sociales del respeto a uno mismo). Al mantener estas ideas, la teoría de Rawls no es adecuada para la consideración de la discapacidad; en primer lugar, porque es excluyente; y como segundo punto, porque no proporciona los verdaderos medios que son necesarios para que una persona en situación de discapacidad pueda autorrealizarse (Cuenca, 2012).

Rawls, siguiendo el modus operandi clásico del contrato social, postula una situación original en la que se negocian los principios de la justicia. Las personas que están en esta negociación son individuos que poseen "poderes morales" plenos, esto es, son sujetos que disponen de racionalidad (la búsqueda de medios para lograr los fines propios) y razonabilidad (comprensión de que las consideraciones personales deben estar en armonía con las de los demás) y que tienen una concepción propia del bien (tienen una idea sobre lo que es tener una vida buena). De manera que los sujetos de la posición original “(...) poseen, al menos, en el grado mínimo esencial, las capacidades morales, intelectuales y físicas que les permiten ser miembros plenamente cooperantes de la sociedad a lo largo de un ciclo de vida completo" (Rawls, 2012, p. 216217). Sin este mínimo de capacidades no existiría manera de entender cómo es que los individuos aceptarían la cooperación, puesto que puede existir la posibilidad de que no todos colaboren de la misma manera, lo cual anularía la motivación de la cooperación (Nussbaum, 2007).

Precisamente, las PcD son aquellas que no cooperarían, porque ni siquiera tienen el mínimo de las capacidades una sociedad individualista requiere (Nussbaum, 2007; Sen, 2014). Así, las personas con deficiencias físicas no estarían integradas, porque es claro que no pueden colaborar de la misma manera que los demás. Al mismo tiempo, al carecer de las características que definen la dignidad humana, tampoco pueden participar en la constitución de los principios de justicia. Las personas con deficiencias físicas y mentales implican un alto coste en su proceso de integración, por lo que no es una buena idea -de acuerdo con Rawls- incluirlos en el momento de decisión de los principios de la justicia (Rawls, 1996). Estas razones llevan a Rawls a negar 
la posibilidad de la participación en la constitución de las bases de la sociedad a las PcD. No los considera sujetos de justicia y solamente serán atendidos en la fase legislativa.

Rawls deja claro que entiende el concepto de "plenamente cooperantes" en un sentido que excluye a las personas con deficiencias físicas y mentales. Por lo tanto, las necesidades especiales de las personas con discapacidades solo serán consideradas una vez diseñada la estructura básica de la sociedad. (Nussbaum, 2007, p.122)

\section{Sen y Nussbaum: capacidades para un nuevo contrato social}

Amartya Sen y Martha Nussbaum son reconocidos por haber puesto en el centro del debate el problema de las personas discapacitadas. Lo hacen sin necesidad de salirse de la visión política liberal que estructuró toda la obra de John Rawls. Su punto de partida es el "enfoque de las capacidades" que han venido trabajando en conjunto desde la década de los ochenta y que ha encontrado gran repercusión en los debates sobre educación y políticas sociales, aunque también ha trascendido lo meramente discursivo para convertirse en propuestas prácticas asumidas por algunos países (Nussbaum, 2016). Sen y Nussbaum se diferencian entre sí debido a que el primero no ofrece una teoría de la justicia al estilo de Rawls, sino solamente una propuesta para medir la calidad de vida que, en el mejor de los casos, como él mismo lo sostiene en La idea de la justicia (2014), se puede convertir en un argumento que servirá de insumo a la discusión en la esfera pública. Nussbaum, por su parte, plantea una teoría normativa (2002), que se sustenta en la tradición de la dignidad de la persona (Kant, 1995), en la afirmación de la necesidad de una redistribución equitativa y en una concepción del bien que se funda, precisamente, en las capacidades.

Al inicio del libro en el que plantea su "idea” de la justicia, Sen, sostiene que tradicionalmente han existido dos alternativas a la hora de proponer marcos teóricos para la comprensión de la justicia social. Por un lado, se encuentran los que proponen el ideal de una sociedad justa (donde se ubicaría La teoría de la justicia de Rawls), un institucionalismo trascendental, en el que se buscan los fundamentos últimos que permitirían distinguir una sociedad justa de otra que no lo es. Por el otro lado, se hallan las posturas, como la de él mismo, que se ocupan de las prácticas que se deben efectuar para evitar más injusticias prevenibles. Este aspecto es, para Sen, lo que la gente realmente hace, en contraposición a lo que se esperaría que harían cuando se le ha dado algunos 
recursos o algunos derechos nominales: "El enfoque de la capacidad es un enfoque general, cuyo foco es la información sobre las ventajas individuales, juzgada desde el punto de vista de la oportunidad y no de un ‘diseño' específico sobre la mejor organización de la sociedad” (2014, p. 262).

Aquello que resulta valioso en la vida de las personas son las cosas que efectivamente pueden, quieren y hacen. Este es el punto de partida para el análisis de Sen. Lo que pretenden los individuos es llevar una vida buena, esto es, una vida valiosa, en la medida en que se despliegan sus capacidades, lo que no se logra exclusivamente a través de los recursos. La concepción redistributiva de Rawls reduce las problemáticas sociales a asuntos de ingresos, promueve la idea de concebir el bienestar a partir del PIB nacional, supone que las personas se realizan únicamente por la posesión de determinados derechos básicos. Pero el enfoque de las capacidades sostiene "trasladar este énfasis en los medios a la oportunidad de cumplir los fines y a la libertad sustantiva de realizar esos fines razonados” (Sen, 2014, p.264).

Sen plantea que es evidente que personas que disponen de recursos pueden no llevar una vida buena, es decir, no pueden convertir sus ventajas en calidad de vida, como es el caso de algunos en situación de discapacidad. La idea de la justicia como redistribución de recursos considera que es suficiente con otorgar unos mínimos materiales para que la gente despliegue sus planes de vida, pero olvida, así, que no se pueden confundir los medios con los fines, que no se trata solamente de tener los derechos sino de ver qué es lo que realmente se puede hacer con ellos (Honneth, 2014).

Para Sen, entonces, una persona discapacitada no encuentra su realización en el simple hecho de poseer derechos en algún código o constitución, sino en los modos en que esos derechos logran hacerse efectivos según los intereses que ellas mismas tienen. El problema central con la discapacidad es la pérdida de libertad, pues, dependiendo de las condiciones sociales, políticas, económicas, físicas y mentales, se puede tener o no un margen mayor de decisión autónoma. Es por esto que resulta sensato pensar que una persona discapacitada necesita de mayores ingresos para poder cumplir sus metas, pero también de una adecuación en los espacios, las comunicaciones, los cuidados, las políticas públicas, en las que se tenga presente la necesidad de ampliar la capacidad de elegir entre alternativas valiosas para los individuos en situación de discapacidad: "Las personas tienen características físicas dispares en relación con la edad, el género, la discapacidad, la morbilidad y otros aspectos, lo cual hace muy diversas sus necesidades. Por ejemplo, una 
persona discapacitada o enferma puede necesitar más ingreso para hacer las mismas cosas elementales que una persona menos afectada con un nivel dado de ingresos" (Sen, 2014, p.285).

A diferencia de Sen, Martha Nussbaum emprende el titánico trabajo de plantear una teoría normativa. Pero, al igual que Rawls, su concepción se mantiene en los límites de un liberalismo político consciente del pluralismo y la multiculturalidad: "El enfoque constituye, pues, una forma de 'liberalismo político', que evidencia su respeto por los ciudadanos y las ciudadanas, absteniéndose de pedirles que se adscriban a ninguna doctrina política exigida sobre una visión religiosa o metafísica particular” (Nussbaum, 2012, p.101)

Parte, igualmente, del "enfoque de las capacidades”, y lo presenta como una alternativa tanto a los universalismos esencialistas (Nussbaum, 2012), como a los problemas más profundos de la teoría de John Rawls referentes a su concepción de la racionalidad, la dignidad y las condiciones de la posición original. El enfoque pretende un cambio en la pregunta central: en vez de preguntar, “¿qué recursos tiene esta persona?”, se pasa a la pregunta: “¿qué pueden ser y hacer las personas?” (Sen, 2014).

Dicho de otro modo, el enfoque concibe cada persona como un fin en sí misma y no se pregunta solamente por el bienestar total o medio, sino también por las oportunidades disponibles para cada ser humano. Está centrado en la elección o en la libertad, pues defiende que el bien crucial que las sociedades deberían promover para sus pueblos es un conjunto de oportunidades... (Nussbaum, 2012, p.38)

Las capacidades son las disposiciones humanas que, al actualizarse (en términosaristotélicos delapotencia alaactualización) llevanalaautorrealización (Aristóteles, Trad. 2008). Profundizando en el análisis de las capacidades realiza una distinción entre capacidades combinadas (condicionadas por el medio sociopolítico), internas (similares al capital cultural de Bourdieu) y las básicas (referentes a las facultades innatas de las personas). Su propuesta es que las naciones reconozcan la necesidad de fomentar e intervenir en la realización de los tres tipos de capacidades para todos los seres humanos. En consecuencia, plantea una lista de diez capacidades fundamentales ${ }^{1}$. Como

1 Las capacidades, tal y como aparecen formuladas, en Mujeres y desarrollo humano (2002, pp.7880), son las siguientes: 1. Vida, 2. Salud corporal, 3. Integridad corporal, 4. Sentidos, imaginación, pensamiento, 5. Emociones, 6. Razón práctica, 7. Afiliación, 8. Otras especies, 9. Capacidad para jugar, 10. Control sobre el entorno de cada uno. 
toda capacidad se actualiza en un funcionamiento, y como no se trata de caer en posturas totalitarias, los gobiernos deberán intervenir en la disponibilidad de las capacidades para todas las personas, sin importar sus fines, esto es, como en el caso de Sen, de lo que se trata es de brindar un campo de libertad fundamentado en las capacidades y en los intereses de cada persona.

Si hay algo por lo que es reconocida Nussbaum, es por la manera en que ha trabajado para que las personas con capacidades de diverso tipo (psicológicas, físicas, etc.) puedan recibir la atención que ameritan en el momento en que se plantean los planes de gobierno o las maneras en que deben ser atendidas. Esta tarea encuentra su motivación precisamente en el olvido sistemático al que la tradición filosófica ha confinado la existencia de las personas con discapacidad. De ahí que, para la filósofa estadounidense, el quid de una teoría de la justicia propuesta en una época posterior a John Rawls debe radicar en la afirmación de la autonomía de los individuos entendida como la superación de las condiciones sociales y políticas (capacidades combinadas) que condenan ciertas vidas a la exclusión: “(...) El objetivo político de todos los seres humanos de una Nación debería ser el mismo: todos y todas deberían superar un cierto nivel umbral de capacidad combinada, entendiendo ese deber no como un funcionamiento obligado, sino como una libertad sustancial para elegir y actuar" (Nussbaum, 2012, p.44).

Es por esta razón que la autora norteamericana propone en Las fronteras de la justicia (Nussbaum, 2007, pp.128-138), las siguientes alternativas: a). frente a los sujetos competitivos que solo ven la cooperación como un medio, antepone la complejidad de la motivación humana (el amor, el respeto, la simpatía); b). frente a la dignidad excluyente kantiana, plantea una concepción de la persona más amplia en la que se incluyen a las personas discapacitadas y también a los animales; y c). frente al reduccionismo de los bienes primarios, propone otra definición del bien: lo bueno es la oportunidad que puede tener el individuo de elegir entre el funcionamiento o no de diversas capacidades.

\section{Honneth: intersubjetividad y discapacidad}

Aunque Axel Honneth no tiene una teoría de la justicia en sentido estricto, presenta una estructura argumentativa alternativa a la discusión en torno a la redistribución, que resulta llamativa cuando se trata de examinar las luchas sociales por la identidad. Lo que se observa en los trabajos de Honneth no es una teoría de la justicia en el sentido de indicaciones para conformar las instituciones sociales y los modos de distribución de los beneficios y las 
cargas, sino la presentación de las condiciones morales sobre las que las instituciones deben ser evaluadas (Honneth, 2014). Honneth, propone que esas condiciones deben sustentarse sobre una concepción social de la libertad en la que las instituciones, a diferencia de lo que ocurre con las libertades negativa y positiva, no se entienden como contrarias ni separadas de aquellas intersubjetivas e institucionales que determinan a los sujetos. Esta afirmación se sustenta sobre una visión de la vida social construida sobre la evidencia empírica en torno a la función central que la interacción posee en la vida de todos, y sobre la propuesta de que la esta interacción debe entenderse como una lucha incesante de tipo moral, que se refiere en concreto a la urgencia de cada individuo por ser reconocido por los demás (Honneth, 1996).

Frente a los problemas de las dos generaciones anteriores de la Teoría Crítica, Honneth propone la idea de una "trascendencia intramundana", esto es, una forma de emancipación que permanezca dentro de las condiciones históricas e institucionales de la razón y que parta de las demandas morales de los sujetos. Así, surge una teoría normativa que toma como materia prima los sentimientos de desprecio de los sujetos y que describe la dinámica de las instituciones en función de la necesidad del reconocimiento mutuo. A esta teoría de los agravios morales como patologías de la razón la sigue una propuesta de emancipación identificada con la lucha por el reconocimiento, es decir, con las acciones que los grupos o los individuos emprenden con el propósito de afirmar su identidad (Honneth, 2009a).

De esta forma, Honneth sostiene que la libertad es y ha sido el fundamento de cualquier concepción de justicia, y que, en consecuencia, la tarea consiste en analizar los tipos de libertad sobre los que se han cimentado las propuestas liberales. La libertad negativa, así como la positiva (o reflexiva) han sido las privilegiadas a la hora de considerar las condiciones necesarias para que los sujetos sean autónomos. Siguiendo la famosa distinción de Isaiah Berlin (2014) se puede describir la libertad negativa como aquella concepción que define la autonomía como la ausencia de obstáculos para el despliegue de los intereses individuales. Mientras que la positiva es la que defiende la idea de una decisión libre de coerciones externas, que enfatiza en el ejercicio de la voluntad y en la capacidad del individuo de darse su propia ley para lograr la autonomía. El primer tipo de libertad es la misma sobre la que los sujetos de la posición original de Rawls deciden los principios de la justicia, y el segundo es el mismo sobre el que se diseña la concepción que destaca la centralidad de lo que hacen los sujetos cuando pretenden desplegar sus capacidades. 
Sin embargo, Honneth, encuentra en estas conceptualizaciones de la libertad algunos inconvenientes (Honneth, 2014). La libertad negativa, como lo han señalado algunos autores de la propia tradición liberal (Manent, 2011), posee el inconveniente de no contar con una guía normativa para su ejercicio; además, reduce la acción del sujeto en la sociedad al cálculo del interés que lleva al beneficio individual. Esto hace del individuo un autómata, que simplemente sigue los mandatos de su deseo, quien desconoce el origen del mismo y niega la interacción con los otros. Dice Honneth: “(...) Siempre es la causalidad, ya sea de la naturaleza interna o de un espíritu anónimo la que dirige al sujeto por detrás de sus espaldas en la elección de sus objetivos de acción” (2014, p. 46). De esta manera, la justicia se ocuparía de brindarle al individuo el margen de acción suficiente, limitado solo por la ley, para que despliegue sus intereses.

Por su parte, la libertad positiva, al enfatizar en la necesidad de decisiones que surjan de la razón individual y que sean el producto del ejercicio pleno sin coerciones de la voluntad, se representa bien en la idea habermasiana de decisiones, que son el producto de la deliberación entre alternativas, a partir de argumentos públicos y que se concretan en un consenso libre. Aunque la propuesta de Honneth no es completamente antagónica a la de Habermas, desde sus primeros trabajos (2009c) señala que las condiciones ideales de comunicación no son un aspecto satisfecho en gran número de contextos actuales, y esto implica que, en sociedades en las que estas condiciones no se encuentren, no es posible proponer la deliberación como el camino hacia la emancipación (Uribe, 2005). Para que exista y se despliegue este libre ejercicio de la voluntad a través de la deliberación se requiere la existencia de las instituciones que posibiliten el despliegue de la intersubjetividad. Esto es muy cercano a la idea hegeliana de la eticidad, en la que no solo es desde el individuo que se realizan los fundamentos éticos de una sociedad, sino desde la interacción con los otros mediada por las instituciones. Por estas razones, la libertad reflexiva es insuficiente para fundar una teoría de la justicia: desconecta al individuo de las condiciones sociales que son necesarias para que pueda ejercer su capacidad deliberativa: “(...) Queda fuera de foco el hecho de que tanto el yo como el nosotros solo podrían ejecutar su autodeterminación si encontraran en la realidad social condiciones institucionales que ofrecieran oportunidad de realización a sus metas” (2014, p. 55). Al final, el fundamento de la justicia desde esta concepción positiva o reflexiva, consiste en brindar las condiciones necesarias para que los individuos se autorrealicen sin perjudicar a los demás, pero olvidando las condiciones sociales de interacción con los otros. 
La propuesta de Honneth es, entonces, un tercer tipo de libertad, que él denomina social y que busca superar los problemas en los que habían caído las dos concepciones anteriores. Esta libertad surge de la teoría central de su propuesta, es decir, de la teoría del reconocimiento. Honneth plantea, en una de sus primeras obras La lucha por el reconocimiento (1995), que la vida social debe ser comprendida, tal y como la había concebido el joven Hegel, como una lucha por el reconocimiento. Esto significa que la sociedad solo puede entenderse de manera contingente, y no como una totalidad cerrada, en la medida en que grupos diversos plantean un conflicto para lograr ser reconocidos. El sociólogo y filósofo de la Escuela de Frankfurt define esta lucha como una teoría normativa de la sociedad, porque indica las formas de autorrealización y las formas de negación de esta.

Honneth parte de la teoría del reconocimiento para criticar a la corriente del liberalismo que se sustenta sobre la defensa de la libertad negativa, porque niega la realidad de la intersubjetividad en aras de la autorrealización. Así, propone su idea de libertad social: la afirmación de la autonomía del individuo, pero teniendo presente que esa autonomía se despliega en las instituciones que constituyen la vida democrática:

(...) 'libre’ es en último término el sujeto solo cuando en el marco de prácticas institucionales se encuentra con una contraparte a la cual lo conecta una relación de reconocimiento mutuo, porque puede ver en las metas de este una condición de la realización de las propias metas. (2014, p.68).

La justicia, desde esta perspectiva, debe fundarse sobre la base de una concepción del sujeto en la que el otro no se entiende como el límite de la acción libre, sino que la intersubjetividad es vista como la condición indispensable de cualquier tipo de reconocimiento, o sea, de cualquier realización de la autonomía. Es así que para Honneth, la justicia debe consistir en la creación de las condiciones institucionales que permitan el ejercicio pleno de la libertad social.

Ahora bien, la teoría del reconocimiento ha resultado de mucha utilidad a la hora de acercarse al problema de la discapacidad. Lo más atractivo del enfoque del reconocimiento tiene que ver con el hecho de privilegiar la perspectiva de los propios afectados, pues, los sentimientos de desprecio surgen de la autopercepción de ellos mismos. De esta manera, no se cae en el error de proponer fórmulas universalistas de inclusión que realmente no dan cuenta de las demandas de las personas en situación de discapacidad. Este es el error, por ejemplo, del redistribucionismo que, al reducir la justicia al otorgamiento 
de derechos, no se ocupa de la especificidad de las limitaciones de las personas en situación de discapacidad. En este sentido, se puede entender la situación social de la discapacidad como una falta de reconocimiento que se evidencia en los ámbitos de la familia, el Estado y la sociedad. Cuando en la propia familia se estigmatiza y se rechaza a la persona discapacitada, se le está negando el amor, está limitada en su autoconfianza; cuando el Estado no reconoce por medio de la Ley el valor de estas personas, no se les está tratando como iguales y ellas pierden su autorrespeto; y finalmente, cuando la sociedad no las integra al mundo laboral, sienten que no tienen ningún mérito, que no aportan nada a la comunidad y, por ende, pierden su autoestima.

Las condiciones que debe cumplir una concepción de justicia, que son propuestas por Honneth, obligan a pensar en los obstáculos que determinadas sociedades pueden llegar a tener para la autorrealización intersubjetiva, en la medida en que desconoce y deja a un lado a miembros que realizan su libertad cuando son reconocidos por los otros. De esta manera, las tareas de una teoría de la justicia - siguiendo en esto a la profesora Ángela Uribe Botero (2005)deben consistir en saber interpretar las demandas de aquellos que no pueden entrar en la defensa y realización de sus derechos por condiciones adversas físicas o mentales. Esta interpretación no puede hacerse de otra manera más que incluyendo -sin reduccionismos ni universalismos- la manera en que los propios sujetos afectados se autoperciben moralmente. Las palabras de la profesora Uribe Botero ilustran de mejor manera este punto:

Incluir al otro sugiere, entonces, no tanto escucharlo para evaluar en qué medida su voz puede ser integrada a un proceso crítico de argumentación cuyo resultado sea el punto de vista objetivo. Incluir al otro sugiere, más bien, situarnos ante él como alguien que escucha asombrado un relato sobre el dolor que causa ver inhibidas las posibilidades de llevar una vida lograda. Una buena manera de sentir que se está en comunicación con alguien que cuenta un relato sobre el dolor no es creyendo que se siente lo que este siente, y por lo tanto, que se entiende lo que él dice, sino más bien y sobre todo, preguntando y estando abierto a las preguntas. (2005, p.103).

\section{Iris Marion Young: discapacidad como transformación política}

Otra teoría de la justicia que termina por afirmar la naturaleza política de la justicia es la de Iris Marion Young (2000). Lo más destacado de su trabajo inicial sobre el problema de la justicia es su enfoque en señalar qué es la injusticia, lo que le permite afirmar que la justica debe entenderse como 
una transformación social en la medida en que lo injusto se encuentra en la estructura del sistema capitalista, que excluye, en razón de la división del trabajo y la industria cultural que defiende, a grupos minoritarios como los pobres y las mujeres.

Su crítica a la redistribución se basa en dos premisas: a). se concentra en la asignación de recursos y desconoce las estructuras sociales que promueven la injusticia; b). convierte a los bienes inmateriales (como los derechos) en cosas cuantificables. Para ella, lo importante en este sentido es estudiar las estructuras de poder que determinan la distribución, pero no la distribución misma, eso sería tomar el efecto por la causa. De la misma manera, al existir demandas sociales que no se agotan en la distribución de recursos, sino que buscan reivindicaciones sociales, como en el caso del feminismo, la justicia no puede obviar la complejidad de la vida social. Las personas no solo buscan que se les asignen recursos, sino que buscan llevar una vida plena en la que su autonomía y autodeterminación pueda desarrollarse de acuerdo a sus capacidades (Young, 2000, p. 30). Para Young, en última instancia, el problema con la redistribución es que no cuestiona la ideología capitalista (v.g. machismo) ni las relaciones de clase. Así, su conclusión es que son el contexto institucional, y todo el aparato ideológico que lo legitima, los responsables de las injusticias ${ }^{2}$ :

Quiero más bien desplazar la discusión sobre la justicia -que toma en cuenta a las personas como las poseedoras y consumidoras principales de los bienes-, hacia un contexto más amplio que incluya también la acción, las decisiones sobre la acción y la provisión de los medios para desarrollar y ejercer las capacidades. (2000, p. 33)

Para Young son tres los ámbitos de la vida humana sobre los que se ejerce la injusticia: en la toma de decisiones, en la división del trabajo y en la cultura. Establecer quién toma las decisiones resulta ser un asunto que puede reproducir las desigualdades; el valor que se le otorga al trabajo es decisivo para establecer quién recibe menos de lo que merece (este puede ser el caso de las personas que cuidan a las personas con discapacidad); $\mathrm{y}$, finalmente, los símbolos y significados que se asocian con determinados grupos son

2 Esto, aunque Young no se compromete con una postura marxista ortodoxa en sentido estricto. En primer lugar, porque las relaciones de clase no son el único problema importante para la justicia (eso sería caer en un economicismo reduccionista); y, en segundo lugar, porque hay aspectos relacionales de la vida en sociedad que no pueden ser objeto de una distribución, sino que ameritan una transformación cultural que se acercaría más a la hegemonía de Gramsci, por ejemplo. 
relevantes a la hora de determinar el valor que pueden llegar a tener para la sociedad (este es el caso del lenguaje y las representaciones sociales que se tienen en torno a las mujeres):

Todos estos factores son relevantes al momento de realizar evaluaciones sobre la justicia, en la medida en que condicionan la aptitud de la gente para participar en la determinación de sus acciones y su aptitud para desarrollar y ejercer sus capacidades. (p. 42)

En últimas, estas injusticias resultan de un contexto institucional que debe entenderse, a la manera de Foucault, como el producto de relaciones de poder sustentadas en dispositivos que no se pueden enfrentar sencillamente con distribuciones que no cuestionan esas mismas relaciones establecidas (Foucault, 2010a). ¿Cómo se puede construir una concepción de justicia sobre este diagnóstico de las injusticias?

La respuesta de Young es esta: "Tal como lo entiendo aquí, el concepto de justicia coincide con el concepto de lo político” (2000, p. 62). Es decir, la justicia es la participación en la constitución del contexto institucional, principalmente, porque la opresión (trabas institucionales al autodesarrollo) y la dominación (trabas institucionales a la autodeterminación) solo pueden ser contrarrestadas a través de la participación democrática. Esto no significa una negación absoluta de la redistribución; significa que la redistribución no debe hacerse sobre criterios establecidos de manera no deliberativa, sino que deben estar sustentados sobre el consenso y la participación de los implicados.

Young, a diferencia de la mayoría de los teóricos de la justicia hasta el momento de la publicación de su trabajo, La justicia y la política de la diferencia (1990), sí se refiere de manera directa al problema de la discapacidad. Esta condición es, para ella, un tipo de "marginación”, que es una subcategoría dentro del problema de la opresión. Define de esta forma la marginación: “... La marginación es injusta porque bloquea las oportunidades de ejercer las capacidades en modos socialmente definidos y reconocidos" (2000, p. 95). Y agrega que "la gente mayor es oprimida a través de la marginación y el imperialismo cultural, y esto sucede también con la gente discapacitada física o mentalmente” (2000, p. 112). Y la marginación en las personas en situación de discapacidad se evidencia en la dependencia para la toma de decisiones colectivas.

La propuesta de Young consiste en ampliar la idea de ser moral, no hacerla depender de las condiciones de la ciudadanía y la racionalidad en sentido 
kantiano: "Una contribución importante de la teoría moral feminista ha sido el cuestionar la presunción, profundamente arraigada, de que el ser moral y la ciudadanía plena requieren que la persona sea autónoma e independiente" (2000, p. 96). Es claro, entonces, que para Young, la marginación de las personas en situación de discapacidad se debe a las restricciones que, desde lo institucional, se han impuesto desde la categoría de persona como condición para ser autónomo y participar en las decisiones en torno a lo común. Pero, al mismo tiempo, la marginación se enfrenta por medio de lo político, a través de una participación efectiva en la constitución de lo común.

El principio de justicia para las PcD debe sustentarse en el acuerdo que pueda llegar a existir alrededor de las políticas que se empleen para la inclusión y atención de ellos. Se trata, entonces, de un criterio político de la justicia.

\section{Conclusión: ¿Cuál modelo de justicia social para las PcD?}

En la Convención... la palabra "participación” es repetida en todo el documento diecinueve veces, y en el Artículo 19 queda sintetizada gran parte de la intención de este documento:

Los Estados Partes en la presente Convención reconocen el derecho en igualdad de condiciones de todas las personas con discapacidad a vivir en la comunidad, con opciones iguales a las de las demás, y adoptarán medidas efectivas y pertinentes para facilitar el pleno goce de este derecho por las personas con discapacidad y su plena inclusión y participación en la comunidad. (Naciones Unidas, 2019)

De manera que el paradigma asistencialista que resultó del modelo de la prescindencia quedó atrás, y ahora la discapacidad se entiende como una formación cultural y una articulación de sentidos y significados contingentes, dependientes de determinaciones culturales e históricas (Barton, 1998). El modelo social se ha constituido en el punto de referencia para cualquier tipo de abordaje estatal alrededor de las PcD. Esto obliga a pensar en la tesis de que la concepción de justicia más acorde con este modelo es aquella en la que se resalten las posibilidades de participación en la constitución de lo común por parte de estas personas. 
¿Cómo entender esta participación política? Existen dos grandes tradiciones de participación: a). La liberal y procedimental (Rawls, 1996), considera que el camino para que los ciudadanos se involucren y tomen partido por lo que se hace con el poder político, se encuentra en los medios institucionales para tal fin, esto es, los derechos políticos. El voto aparece como la práctica por excelencia de este modelo, aunque también se refiere a la formación de grupos, a la participación activa en partidos y al ejercicio del control político por medio del voto o de la oposición argumentada; b). La lucha política o emancipación, donde la participación, según esta tradición, sucede más allá del ejercicio electoral e institucional y se concentra en las prácticas micropolíticas en las que las personas entran en su cotidianidad por medio de su oposición a determinadas formas de vida o esquemas de asimilación de la diferencia de carácter simbólico. Un buen ejemplo de este tipo de participación es el de Rosa Parks, quien en 1995 se negó a cederle la silla del autobús a un blanco (siendo ella negra), lo que claramente, en ese momento, se constituyó en un caso de oposición con respecto a los marcos culturales y políticos de referencia. Este es un caso de participación política "paraestatal", que se convirtió en una transformación social con repercusiones en todo el planeta. Acá, los significados y los sentidos sociales fueron trastocados por medio de una acción que no se concentró en los canales representativos e institucionales de participación.

Esta necesidad de transformación puede comprenderse mejor si nos referimos al contexto colombiano cuando se revisan las observaciones de la ONU al Informe sobre la situación de los derechos de las personas con discapacidad en Colombia del 31 de agosto de 2016, en el que Colombia presentó unos resultados pobres frente a los objetivos propuestos en el marco de la Convención. En especial, en el aspecto referente a la toma de conciencia, en el artículo 8, se consigna lo siguiente: "El comité observa con preocupación que las campañas de "sensibilización”, públicas y privadas, promovidas sobre las personas con discapacidad, tal como el Teletón y la celebración del Día Blanco reflejan el modelo caritativo de la discapacidad". Y en el artículo 29, en el numeral 64, se indica que: "Al Comité le preocupan las restricciones a la participación política de personas con discapacidad, particularmente que las personas declaradas interdictas no pueden ejercer su derecho al voto y que no se garantice la accesibilidad en procesos electorales”. Estas alertas hablan de una nula asimilación del modelo social de la discapacidad; pero también de una exclusión radical de la posibilidad de tener autonomía política y poder tomar decisiones que constituyan lo común. Resulta consecuente pensar que la justicia debe entenderse, en este contexto, como el ejercicio del poder político por parte de las PcD en el que se presente una reactivación de la sociedad que 
permita su inclusión. De modo que la justicia se referirá a las posibilidades de participar en la toma de decisiones comunes, es decir, a la efectividad de transformación social que puedan lograr las PcD.

No se puede negar que, tras la Convención, y el cambio de paradigma que implicó su adopción en más de 163 Estados, se han obtenido diversos avances en materia de participación política por parte de las PcD. En septiembre de 2015, la discapacidad hace parte de los 17 objetivos de desarrollo sostenible de las Naciones Unidas, pero, particularmente se señala como un propósito ineludible el hecho de promover la inclusión, social, económica y política.

En algunos países europeos se ha abierto la puerta para que las personas con discapacidad intelectual puedan participar en la política, bien sea como votantes o representantes. Es el caso de lo ocurrido en 2018 en España, donde a 100000 personas con este tipo de discapacidad se les ha permitido llevar a la práctica sus derechos políticos. Esto se logró gracias a una reforma promovida por el sector progresista del espectro político, en la que la Ley Orgánica de Régimen Electoral General (LOREG) fue modificada para dar paso a la materialización de los derechos políticos de este sector de la sociedad. Se trató de un trabajo conjunto entre la sociedad civil (liderada por miembros de la comunidad de personas con discapacidad intelectual) y el Partido Socialista Español (PSOE), lo que llevó, al final, a la adopción unánime de la modificación en la ley electoral por parte del Congreso.

Pero, sin duda, en este caso, y tal y como lo manifiestan los impulsores de la reforma, que iban desde miembros de grupos de discapacitados hasta políticos comprometidos con esta problemática, "es un éxito democrático, vamos a hacer historia madurando la democracia española” (Marcos, 2018). Estas transformaciones han resultado en una realidad gracias a la presión de los sectores marginados y a la exposición de demandas frente a la institucionalidad, que se hacen cada vez más imperiosas de resolver, debido a la expansión de los derechos que surgen de la Convención y que buscan ser realizados en la práctica de cada país, cada comunidad, cada región, cada grupo específico. Como bien lo dicen los promotores de la reforma, “...hemos redoblado la presión política” (Marcos, 2018), de otra forma, la marginación y exclusión se mantendrían.

En el caso colombiano se tienen diferentes marcos normativos que constituyen un avance en el reconocimiento de los derechos políticos de las PcD. El capítulo II de la Ley 1618 de 2013 es el hito en lo referente a 
participación política. Los artículos 22, 23, 24, 25 y 26 plantean las líneas principales de los límites y alcances de la participación política de las PcD. Los artículos se basan en la Ley 134 de 1994, en la que se da cuenta de las maneras de acceso a la institucionalidad política que tienen los colombianos, incluidos las PcD, con lo que queda claro aquello que se ha evidenciado en el informe de 2016 acerca de la ausencia de una especificidad en relación con las necesidades, medios y capacidad de decisión que pueden llegar a tener los individuos pertenecientes este grupo social en el marco del ejercicio de la participación política. En la ley se señala al control social, la inclusión de las mujeres, la elaboración de políticas públicas y la evaluación del cumplimiento de los compromisos de la Convención como las cuestiones centrales por tener presentes a la hora de establecer la inclusión política de las PcD.

A diferencia del caso español, en Colombia no existe una reforma electoral enfocada en las necesidades y demandas de las PcD. En la Ley 163 de 1994 se abre la posibilidad de que, en caso de ser necesario, se cuente con un acompañante a la hora de votar por parte de estas personas. Pero tanto la falta de un enfoque diferencial como la reducción de la participación política al momento electoral, hablan de una visión de la discapacidad en la que se niega la necesidad de un tipo de justicia, entendida como autonomía en la toma de decisiones acerca de lo que afecta a la comunidad. Por el contrario, el informe de evaluación de la aplicación de la Convención en Colombia revela el carácter reduccionista con el que el Estado asume el imperativo de la inclusión política que se exige desde este sector de la sociedad. Es por esto que un modelo alternativo de justicia social contribuye, de manera discursiva, para lograr una inclusión acorde con los términos que los propios afectados demandan.

Haciendo un balance de las teorías que se han revisado, se encuentra que las teorías redistribucioncitas, como las de Rawls, Nussbaum y Sen, se enfocan en las maneras en que el Estado puede proporcionar, a través de canales institucionales, la satisfacción de las demandas de las personas, bien sean en lo relativo a los derechos o bien a las posibilidades para desplegar las capacidades. Son planteamientos éticopolíticos que parten del supuesto -en el caso de Rawls- de sociedades iguales y libres en las que los derechos básicos son garantizados para todos los miembros de la Nación; y en el caso de Nussbaum y Sen, se asume la justicia desde el paradigma del individualismo y la homogeneidad universal del ser humano.

Iris Marion Young invita a entender la justicia como una acción política en la que los marginados de la sociedad exigen su reconocimiento como iguales 
y plantean la necesidad de una reconfiguración de lo común a partir de sus propias demandas. Esta es una concepción de justicia que, cuestionando la línea redistributiva, se concentra en los aspectos culturales y simbólicos de la exclusión social. Para Young, la justicia tiene que marcar una ruptura con los símbolos y significados que han constituido una sociedad en la que determinados grupos son excluidos e invisibilizados. Esta teoría de lo político sostiene que solo puede existir justicia en la medida en que los individuos afectados históricamente puedan participar políticamente para reconstituir la sociabilidad, los vínculos y el lenguaje sobre el que se ha cimentado su exclusión y marginación.

Finalmente, Axel Honneth proporciona una teoría normativa en la que se pueden identificar las pretensiones de reconocimiento a partir de las mismas autopercepciones de los afectados. Por medio de una descripción del modo en que se perciben a sí mismos, según su sentimiento de inclusión, de autorrespeto o autoestima, la teoría normativa de Honneth permite comprender los problemas de exclusión escuchando la voz de los individuos en cuestión, sin imponer modelos preconcebidos de derechos o de desarrollo de capacidades. Propone, además, un vínculo profundo entre la autorrealización, la intersubjetividad y las instituciones; esto es relevante a la hora de pensar en la justicia para las PcD, puesto que indica la ineludibilidad de factores emocionales, sociales y políticos que son necesarios para la realización de cualquier idea de bien.

Por último, cuando las teorías de la justicia se refieren a la discapacidad, adoptan una posición dependiendo de lo que se considere el problema de la injusticia: distribución, marginación o desprecio. En consecuencia, la propuesta de justicia social se referirá a las condiciones que se deben cumplir para la realización del ideal. Pero, cuando se analizan a la luz de un contexto específico como el de la PcD en Colombia, la concepción más persuasiva es aquella que brinda una posibilidad de transformación de las estructuras institucionales y culturales que promueven la marginación, la injusticia.

\section{Referencias}

Aristóteles. (2007). Ética a Nicómaco. Madrid: Alianza.

Barton, L. (Comp.) (1998). Discapacidad y sociedad. Madrid: Morata.

Berlin, I. (2014). Las ideas políticas en la era romántica. México: Fondo de Cultura Económica. 
Organización de las Naciones Unidas (ONU). (2006). Convención sobre los Derechos de las Personas con Discapacidad. Recuperado de:. https://www.un.org/esa/socdev/ enable/documents/tccconvs.pdf

Cuenca Gómez, P. (2012). Sobre la inclusión de la discapacidad en la teoría de los derechos humanos.Revista de Estudios Políticos CEPC, N. ${ }^{\circ 158}$, oct-dic, pp. 103-137.

De Asís Roig, R.(2006). Derechos humanos y discapacidad. En Jiménez, E. (Coord.) Igualdad, no discriminación y discapacidad. Buenos Aires: Ediar-Dykinson.

FOUCAULT, M. (2010a). Historia de la sexualidad I.Buenos Aires: Siglo XXI.

FOUCAULT, M. (2010b). Vigilar y castigar. México: Siglo XXI.

Fraser, N.(2010). Escalas de justicia. España: Herder.

Fraser, N., Honneth, A. (2006). ¿Redistribución o reconocimiento? Un debate políticofilosófico. Madrid: Morata.

Grueso, D. I. (2010). La especificidad filosófico-política de la pregunta por la justicia. Coherencia, Vol.7, N. ${ }^{\circ} 13$. pp. 181-211.

Hobsbawn, E. (2015). Historia del siglo XX. Madrid: Crítica.

Honneth, A. (2009c). Crítica del poder: fases en la reflexión de una teoría crítica de la sociedad. Madrid: Machado

Honneth, A. (2014). El derecho de la libertad. Buenos Aires: Katz.

Honneth, A. (1996). La lucha por el reconocimiento. Barcelona: Grijalbo-Mondadori.

Honneth, A. (2009b). Crítica del agravio moral. Buenos Aires: Fondo de Cultura Económica.

Honneth, A. (2009a). Patologías de la razón. Historia y actualidad de la teoría crítica. Buenos Aires: Katz.

Manent, P. (2011). Grandeza y miseria del liberalismo. Cuadernos de Pensamiento Político, N. ${ }^{\circ} 30$, pp.55-67.

Marcos, J. (17 de octubre de 2018). El Congreso reconoce el derecho a votar de 100.000 discapacitados intelectuales. El País. Recuperado de: https://elpais.com/ politica/2018/10/17/actualidad/1539804297_438797.html

Martínez-Rozo, A. M., Uribe-Rodríguez, A. F., Velázquez-González, H. J. (2014). La discapacidad y su estado actual en la legislación colombiana. Revista de la Facultad de Ciencias de la Salud, Vol.12, N.¹, pp.49-58.

Mcintyre, A.(2001). Tras la virtud. Barcelona: Crítica.

Ministerio de Salud y Protección Social de Colombia (2017). Sala situacional de las personas con discapacidad (PCD), tomado de; https://www.minsalud.gov.co/ sites/rid/Lists/BibliotecaDigital/RIDE/DE/PES/presentacion-sala-situacionaldiscapacidad-2017.pdf, consultado el 25 de noviembre de 2019.

Mouffe, C. (2010). En torno a lo político. Buenos Aires: Fondo de Cultura Económica.

Nussbaum, M. (2012). Crear capacidades. Barcelona: Paidós.

Nussbaum, M. (2007). Fronteras de la justicia. Barcelona: Paidós.

Nussbaum, M. (2002). Las mujeres y desarrollo humano. Barcelona: Herder.

Organización Mundial de la Salud (2017). 10 datos sobre la discapacidad, https://www. who.int/features/factfiles/disability/es/, consultado el 25 de noviembre de 2019

Padilla-Muñoz, A. (2010). Discapacidad: contexto, concepto y modelos., International Law: Revista Colombiana de Derecho Internacional, N. ${ }^{\circ} 16$, Ene-Jun, pp.381-414.

Palacios, A. (2008). El modelo social de la discapacidad: orígenes, clasificación y plasmación. Madrid:Cinca. 
Palacios, A. (2017). El modelo social de discapacidad y su concepción como cuestión de derechos. Revista Colombiana de Ciencias Sociales, Vol.8, N. ${ }^{\circ} 1$, pp.14-18.

Palacios, A. y Bariffi, F. (2007). La discapacidad como cuestión de derechos humanos. Madrid: Cinca.Petrucciani, S. (2010). Modelos de filosofía política. Buenos Aires: Amorrortu.

Rawls, J. (1996). Liberalismo político. Madrid: Crítica.

Rawls, J. (2012). Teoría de la justicia. Ciudad de México: Fondo de Cultura Económica. Sandel, M. (2000). El liberalismo y los límites de la justicia. Barcelona: Gedisa.

Schmitt, C.(2006). Legalidad y legitimidad. España: Comares.

Sen, A. (2014). La idea de la justicia. Bogotá: Taurus.

Uribe Botero, Á. (2005). Reconocimiento y humildad: lo que media en la inclusión del otro. Revista Internacional de Filosofía Política, N.²6, pp. 89-106.

Young, I. M. (2000). La justicia y la política de la diferencia. Madrid:Cátedra. 